\title{
ERRATUM: LINEAR PROJECTIONS AND SUCCESSIVE MINIMA
}

\author{
CHRISTOPHE SOULÉ
}

\section{§1. Erratum}

The proof of Proposition 1 and Theorem 2 in [3] is incorrect. Indeed, Sections 2.5 and 2.7 in [3] contain a vicious circle: the definition of the filtration $V_{i}, 1 \leq i \leq n$, in Section 2.5 of that article depends on the choice of the integers $n_{i}$, when the definition of the integers $n_{i}$ in Section 2.7 depends on the choice of the filtration $\left(V_{i}\right)$. Thus, only Theorem 1 and Corollary 1 in [3] are proved. In the following we will prove another result instead of [3, Proposition 1].

\section{$\S 2$. An inequality}

2.1. Let $K$ be a number field, let $O_{K}$ be its ring of algebraic integers, and let $S=\operatorname{Spec}\left(O_{K}\right)$ be the associated scheme. Consider a Hermitian vector bundle $(E, h)$ over $S$. Define the $i$ th successive minima $\mu_{i}$ of $(E, h)$ as in [3, Section 2.1]. Let $X_{K} \subset \mathbb{P}\left(E_{K}^{\vee}\right)$ be a smooth, geometrically irreducible curve of genus $g$ and degree $d$. We assume that $X_{K} \subset \mathbb{P}\left(E_{K}^{\vee}\right)$ is defined by a complete linear series on $X_{K}$ and that $d \geq 2 g+1$. The rank of $E$ is thus $N=d+1-g$. Let $h\left(X_{K}\right)$ be the Faltings height of $X_{K}$ (see [3, Section 2.2]).

For any positive integer $i \leq N$, we define the integer $f_{i}$ by the formulas

$$
\begin{aligned}
& f_{i}=i-1 \quad \text { if } i-1 \leq d-2 g \\
& f_{i}=i-1+\alpha \quad \text { if } i-1=d-2 g+\alpha, 0 \leq \alpha \leq g
\end{aligned}
$$

Received July 27, 2012. Revised May 29, 2013. Accepted October 30, 2013.

First published online December 9, 2014.

2010 Mathematics Subject Classification. Primary 14G40; Secondary 14H99, 11H06. 
Fix two natural integers $s$ and $t$ and suppose that $2 \leq s<t \leq N-2$. When $2 \leq i \leq s$, we let

$$
A_{i}=\frac{f_{i}^{2}}{(i-1) f_{i}-\sum_{j=2}^{i-1} f_{j}}
$$

and, when $t \leq i \leq N$,

$$
A_{i}=\frac{f_{i}^{2}}{\left((i-t+s) f_{i}-\left(f_{1}+f_{2}+\cdots+f_{s}+f_{t}+\cdots+f_{i-1}\right)\right)}
$$

(with the convention that $f_{t}+\cdots+f_{t-1}=0$ ). Consider

$$
A(s, t)=\max _{2 \leq i \leq s \text { or } t \leq i \leq N} A_{i} .
$$

THEOREM 1. There exists a constant $c(d)$ such that the following inequality holds:

$$
\begin{aligned}
& \frac{h\left(X_{K}\right)}{[K: \mathbb{Q}]}+(2 d-A(s, t)(N-t+s+1)) \mu_{1} \\
& \quad+A(s, t)\left(\sum_{\alpha=1}^{N+1-t} \mu_{\alpha}+\sum_{\alpha=N+1-s}^{N} \mu_{\alpha}\right)+c(d) \geq 0 .
\end{aligned}
$$

2.2. To prove Theorem 1 , we start by the following variant of Corollary 1 in $[1]$.

Proposition 1. Fix an increasing sequence of integers $0=e_{1} \leq e_{2} \leq$ $\cdots \leq e_{N}$ and a decreasing sequence of numbers $r_{1} \geq r_{2} \geq \cdots \geq r_{N}$. Assume that $e_{s}=e_{s+1}=\cdots=e_{t-1}$ and that $e_{i-1}<e_{i}$ when $i \leq s$ or $i \geq t$. Let

$$
S=\min _{1=i_{0}<\cdots<i_{\ell}=N} \sum_{j=0}^{\ell-1}\left(r_{i_{j}}-r_{i_{j+1}}\right)\left(e_{i_{j}}+e_{i_{j+1}}\right) .
$$

Then

$$
S \leq B(s, t)\left(\sum_{j=1}^{s}\left(r_{j}-r_{N}\right)+\sum_{j=t}^{N}\left(r_{j}-r_{N}\right)\right)
$$

where

$$
B(s, t)=\max _{2 \leq i \leq s \text { or } t \leq i \leq N} B_{i},
$$

and $B_{i}$ is defined by the same formula as $A_{i}$, each $f_{j}$ being replaced by $e_{j}$. 
Proof. We can assume that $r_{N}=0$. As in [1, proof of Theorem 1], we may first assume that $S=1$ and seek to minimize $\sum_{j=1}^{s} r_{j}+\sum_{j=t}^{N} r_{j}$. If we graph the points $\left(e_{j}, r_{j}\right), S / 2$ is the area under the Newton polygon they determine in the first quadrant. Moving the points not lying on the polygon down onto it only reduces $\sum_{j=1}^{s} r_{j}+\sum_{j=t}^{N} r_{j}$, so we may assume that all the points actually lie on the polygon. In particular, we assume that the point $\left(e_{j}, r_{j}\right)=\left(e_{s}, r_{j}\right)$ lies on this polygon when $s \leq j \leq t-1$. For such $r_{i}$ 's we have

$$
S=\sum_{i=1}^{N-1}\left(r_{i}-r_{i+1}\right)\left(e_{i}+e_{i+1}\right) .
$$

Let $\sigma_{i}=r_{i-1}-r_{i}, i=2, \ldots, N$. The condition that the points $\left(e_{i}, r_{i}\right)$ lie on their Newton polygon and that the $r_{i}$ decrease becomes, in terms of the $\sigma_{i}$,

$$
\frac{\sigma_{2}}{e_{2}-e_{1}} \geq \frac{\sigma_{3}}{e_{3}-e_{2}} \geq \cdots \geq \frac{\sigma_{s}}{e_{s}-e_{s-1}} \geq \frac{\sigma_{t}}{e_{t}-e_{t-1}} \geq \cdots \geq 0
$$

Furthermore

$$
\sigma_{s+1}=\cdots=\sigma_{t-1}=0 .
$$

Next, we impose the constraint $\sum_{j=1}^{s} r_{j}+\sum_{j=t}^{N} r_{j}=1$, that is,

$$
\sum_{j=2}^{s}(j-1) \sigma_{j}+\sum_{j=t}^{N}(j-t+s) \sigma_{j}=1
$$

(recall that $\left.r_{N}=0\right)$. In the subspace of the points $\sigma=\left(\sigma_{2}, \ldots, \sigma_{s}, \sigma_{t}, \ldots, \sigma_{N}\right)$ defined by (2), the inequalities (1) define a simplex. The linear function

$$
S=\sum_{2 \leq j \leq s} \sigma_{j}\left(e_{j-1}+e_{j}\right)+\sum_{t \leq j \leq N} \sigma_{j}\left(e_{j-1}+e_{j}\right)
$$

must achieve its maximum on this simplex at one of the vertices, that is, a point where, for some $i$ and $\alpha$, we have

$$
\alpha=\frac{\sigma_{2}}{e_{2}-e_{1}}=\cdots=\frac{\sigma_{i}}{e_{i}-e_{i-1}}>\frac{\sigma_{i+1}}{e_{i+1}-e_{i}}=\cdots=0 .
$$

We get

$$
\sigma_{j}= \begin{cases}\alpha\left(e_{j}-e_{j-1}\right) & \text { if } j \leq i \\ 0 & \text { otherwise }\end{cases}
$$


Then, using (2), we get, if $i \leq s$,

$$
\alpha=\left((i-1) e_{i}-\sum_{j=2}^{i-1} e_{j}\right)^{-1}
$$

and, when $i \geq t$,

$$
\alpha=\left((i-t+s) e_{i}-e_{1}-e_{2}-\cdots-e_{s}-e_{t}-\cdots-e_{i-1}\right)^{-1}
$$

Since

$$
S=\alpha \sum_{j=2}^{i}\left(e_{j}^{2}-e_{j-1}^{2}\right)=\alpha e_{i}^{2}
$$

Proposition 1 follows.

2.3. We come back to the situation of Theorem 1. For every complex embedding $\sigma: K \rightarrow \mathbb{C}$, the metric $h$ defines a scalar product $h_{\sigma}$ on $E \otimes_{O_{K}} \mathbb{C}$. If $v \in E$, we let

$$
\|v\|=\max _{\sigma} \sqrt{h_{\sigma}(v, v)}
$$

Choose $N$ elements $x_{1}, \ldots, x_{N}$ in $E$, linearly independent over $K$ and such that

$$
\log \left\|x_{i}\right\|=\mu_{N-i+1}, \quad 1 \leq i \leq N .
$$

Let $y_{1}, \ldots, y_{N} \in E_{K}^{\vee}$ be the dual basis of $x_{1}, \ldots, x_{N}$. Let $A(d)$ be the constant appearing in [3, Theorem 1]. From [3, Corollary 1], we deduce the following.

LEMmA 1. Assume that $1 \leq s \leq t \leq N-2$. We may choose integers $n_{i}$, $s+1 \leq i \leq t-1$, such that the following holds.

(i) For all $i,\left|n_{i}\right| \leq A(d)+d$.

(ii) Let $w_{i}=y_{i}$ if $1 \leq i \leq s$ or $t \leq i \leq N$, and let $w_{i}=y_{i}+n_{i} y_{i+1}$ if $s+1 \leq$ $i \leq t-1$. Let $\left\langle w_{1}, \ldots, w_{i}\right\rangle \subset E_{K}^{\vee}$ be the subspace spanned by $w_{1}, \ldots, w_{i}$, and

$$
W_{i}=E_{K}^{\vee} /\left\langle w_{1}, \ldots, w_{i}\right\rangle
$$

$\left(W_{0}=E_{K}^{\vee}\right)$. Then, when $s+1 \leq i \leq t-1$, the linear projection from $\mathbb{P}\left(W_{i-1}\right)$ to $\mathbb{P}\left(W_{i}\right)$ does not change the degree of the image of $X_{K}$. 
2.4. Let $\left(v_{i}\right) \in E_{K}^{N}$ be the dual basis of $\left(w_{i}\right)$. We have

$$
v_{i}=x_{i} \quad \text { when } i \leq s+1 \text { or } i \geq t+1
$$

and

$$
v_{i}=x_{i}-n_{i-1} x_{i-1}+n_{i-1} n_{i-2} x_{i-2}-\cdots \pm n_{i-1} \cdots n_{s+1} x_{s+1}
$$

when $s+2 \leq i \leq t$.

From these formulas it follows that there exists a positive constant $c_{1}(d)$ such that

$$
\log \left\|v_{i}\right\| \leq r_{i}= \begin{cases}\mu_{N+1-i}+c_{1}(d) & \text { if } i \leq s \text { or } i \geq t+1 \\ \mu_{N-s}+c_{1}(d) & \text { if } s+1 \leq i \leq t\end{cases}
$$

Let $d_{i}$ be the degree of the image of $X_{K}$ in $\mathbb{P}\left(W_{i}\right)$, and let $e_{i}=d-d_{i}$. By Lemma 1, we have

$$
e_{s}=e_{s+1}=\cdots=e_{t-1} .
$$

Therefore we can argue as in [2, Theorem 1] and [3, pp. 50-53] to deduce Theorem 1 from Proposition 1.

Acknowledgments. I thank Jean-Benoît Bost, Carlo Gasbarri, and Claire Voisin for their help.

\section{REFERENCES}

[1] Morrison, I., Projective stability of ruled surfaces, Invent. Math. 56 (1980), 269-304. MR 0561975. DOI 10.1007/BF01390049.

[2] Soulé, C., Successive minima on arithmetic varieties, Compos. Math. 96 (1995), 85-98. MR 1323726.

[3] - Linear projections and successive minima, Nagoya Math. J. 197 (2010), 45-57. MR 2649279.

Centre National de la Recherche Scientifique and Institut des Hautes Études Scientifiques

91440 Bures-sur-Yvette

France

soule@ihes.fr 